\title{
An Underlying Common Factor, Influenced by Genetics and Unique Environment, Explains the Covariation Between Major Depressive Disorder, Generalized Anxiety Disorder, and Burnout: A Swedish Twin Study
}

\author{
Lisa Mather, ${ }^{1}$ Victoria Blom, ${ }^{1,2,3}$ Gunnar Bergström, ${ }^{4,5}$ and Pia Svedberg ${ }^{1}$ \\ ${ }^{1}$ Division of Insurance Medicine, Department of Clinical Neuroscience, Karolinska Institutet, Stockholm, Sweden \\ ${ }^{2}$ The Swedish School of Sport and Health Sciences, Stockholm, Sweden \\ ${ }^{3}$ Department of Psychology, Stockholm University, Stockholm, Sweden \\ ${ }^{4}$ Division of Intervention and Implementation Research, Institute of Environmental Medicine, Karolinska Institutet, \\ Stockholm, Sweden \\ ${ }^{5}$ Centre for Occupational and Environmental Medicine, Stockholm County Council, Stockholm, Sweden
}

\begin{abstract}
Depression and anxiety are highly comorbid due to shared genetic risk factors, but less is known about whether burnout shares these risk factors. We aimed to examine whether the covariation between major depressive disorder (MDD), generalized anxiety disorder (GAD), and burnout is explained by common genetic and/or environmental factors. This cross-sectional study included 25,378 Swedish twins responding to a survey in 2005-2006. Structural equation models were used to analyze whether the trait variances and covariances were due to additive genetics, non-additive genetics, shared environment, and unique environment. Univariate analyses tested sex limitation models and multivariate analysis tested Cholesky, independent pathway, and common pathway models. The phenotypic correlations were $0.71(0.69-0.74)$ between MDD and GAD, $0.58(0.56-0.60)$ between MDD and burnout, and $0.53(0.50-0.56)$ between GAD and burnout. Heritabilities were $45 \%$ for MDD, $49 \%$ for GAD, and 38\% for burnout; no statistically significant sex differences were found. A common pathway model was chosen as the final model. The common factor was influenced by genetics (58\%) and unique environment $(42 \%)$, and explained $77 \%$ of the variation in MDD, $69 \%$ in GAD, and $44 \%$ in burnout. GAD and burnout had additive genetic factors unique to the phenotypes (11\% each), while MDD did not. Unique environment explained $23 \%$ of the variability in MDD, $20 \%$ in GAD, and $45 \%$ in burnout. In conclusion, the covariation was explained by an underlying common factor, largely influenced by genetics. Burnout was to a large degree influenced by unique environmental factors not shared with MDD and GAD.
\end{abstract}

Keywords: major depressive disorder, anxiety disorders, psychological stress, twins, behavioral genetics

Mental disorders such as depression and anxiety are one of the main reasons for the increase of years lived with disability globally (Global Burden of Disease Study 2013 Collaborators, 2015). Burnout has been found to be closely related to depression (Bianchi et al., 2015a) and has also been found to be associated with anxiety (Ding et al., 2014; Toker et al., 2005). Burnout has been defined as 'A state of physical, emotional, and mental exhaustion caused by long-term involvement in situations that are emotionally demanding' (Pines \& Aronson, 1988, p. 9). There is an ongoing discussion about whether burnout is a unique condition or a form of depression (Bianchi et al., 2015b). Several twin studies have found that depression and anxiety are highly comorbid due to the fact that they share genetic risk factors (Middeldorp, Cath et al., 2005). However, less is known about the genetic risk factors for burnout and whether they are shared with depression and anxiety.

RECEIVED 24 April 2016; ACCEPTED 6 July 2016. First published online 13 September 2016.

ADDRESS FOR CORRESPONDENCE: Lisa Mather, Division of Insurance Medicine, Department of Clinical Neuroscience, Karolinska Institutet, Berzelius väg 3, SE-171 77 Stockholm, Sweden. E-mail: lisa.mather@ki.se 
Burnout has mainly been described as a work-related phenomenon with three dimensions: exhaustion, cynicism, and a sense of ineffectiveness (Maslach et al., 2001). However, measurement instruments have been developed that measure burnout both among persons who are working and among persons without paid work. The Pines Burnout Measure takes a wider perspective on burnout and can be used also in non-working populations (Pines et al., 1981). The Pines Burnout Measure correlates mainly with the exhaustion dimension of the Maslash Burnout Inventory (Shirom \& Ezrachi, 2003).

Major depressive disorder (MDD) and generalized anxiety disorder (GAD) are heritable, and meta-analyses of twin studies have estimated the heritabilities to be $37 \%$ and $32 \%$, respectively (Hettema et al., 2001; Sullivan et al., 2000). Previous twin studies have found a complete overlap between the genetic risk factors for depression and anxiety in women and a very large overlap in men (Kendler et al., 1992; Kendler et al., 2007). Candidate genes have also been found for both depression and anxiety, and the 5-HTTLPR short variant, involved in the serotonergic system was found to be involved in both depression and anxiety disorders (Gatt et al., 2015). The heritability of burnout is less studied. The few studies presented so far have shown inconsistent results, and the heritability was estimated to be $13-37 \%$ (Blom et al., 2012; Middeldorp et al., 2006). The variation in results may be due to the fact that there exists no generally accepted definition of burnout and hence different measurements have been used. In a previous study, we identified that burnout predicted sick leave due to mental disorders, such as depression and anxiety, because of shared genetic risk factors (Mather, Bergström et al., 2014). Another study found that the correlation between burnout and anxious depression was partly explained by genetic factors in common to both (Middeldorp et al., 2006). Moreover, both personal history, as well as a family history of depression have been found to predict emotional exhaustion, the key feature in burnout (Nyklicek \& Pop, 2005), a finding that supports a shared genetic vulnerability.

The aim of this study was to examine to what degree the covariation between MDD, GAD, and burnout is explained by common genetic and environmental factors, in women and men, using a biometric twin design in a large sample of Swedish twins.

\section{Materials and Methods}

\section{Sample}

This study has a cross-sectional design and used data from the Swedish Twin Registry (STR). The STR is a populationbased registry that contains all twins born in Sweden (Magnusson et al., 2013). Twins that responded to the Study of Twin Adults-Genes and Environment (STAGE), performed by the STR in 2005-2006, were included. STAGE was a large web-based questionnaire sent to all twins in the STR born between 1959 and $1985(N=42,582)$, with a response rate of $59.6 \%$. All respondents were included and hence the sample contained 25,378 twins. In the sample, there were 8,646 complete twin pairs, where 2,151 were monozygotic (MZ) females, 1,402 MZ males, 1,510 dizygotic (DZ) females, 1,000 DZ males, and 2,583 DZ opposite sex twin pairs. The sample also contained 8,086 single twins that were included in the analyses. More information about STAGE is available elsewhere (Furberg et al., 2008; Lichtenstein et al., 2006). The mean age was 33.6 (SD 7.7) years and the sample contained $55.6 \%$ women (Table 1 ). The prevalence of MDD, GAD, and burnout did not vary much with age; hence, age was not included in the analyses.

\section{Measures}

Lifetime prevalence of depression was measured with 38 questions based on the Structured Clinical Interview for DSM-IV Disorders (SCID; First et al., 1996). SCID is based on criteria for MDD in the Diagnostic and Statistical Manual of Mental Disorders, Fourth Edition (DSM-IV; American Psychiatric Association, 2000). Criteria A, C, and E had to be fulfilled in order for the participant to be classified as having had MDD. At least five of the following symptoms had to have been present during the same 2-week period; at least one of the symptoms had to be (1) depressed mood or (2) loss of interest or pleasure (criteria A).

1. depressed mood most of the day, nearly every day;

2. markedly diminished interest or pleasure in all, or almost all, activities most of the day, nearly every day;

3. significant weight loss when not dieting or weight gain, or decrease or increase in appetite nearly every day;

4. insomnia or hypersomnia nearly every day;

5. psychomotor agitation or retardation nearly every day;

6. fatigue or loss of energy nearly every day;

7. feelings of worthlessness or excessive or inappropriate guilt nearly every day;

8. diminished ability to think or concentrate, or indecisiveness, nearly every day;

9. recurrent thoughts of death (not just fear of dying), recurrent suicidal ideation without a specific plan, or a suicide attempt or specific plan for committing suicide.

The symptoms had to cause clinically significant distress or impairment in social, occupational, or other important areas of functioning (criteria $\mathrm{C}$ ) and not be better accounted for by bereavement (criteria E; American Psychiatric Association, 2000).

Lifetime prevalence of anxiety was measured with 23 questions based on SCID (First et al., 1996). Criteria A and $\mathrm{C}$ had to be present in order for the participant to be classified as having had GAD. Excessive anxiety and worry, occurring more days than not for at least 6 months, about a number of events or activities had to be reported (criteria A) and the anxiety and worry had to be associated with three or more of the following symptoms (criteria C): 


\begin{tabular}{|c|c|c|c|}
\hline \multicolumn{4}{|c|}{$\begin{array}{l}\text { Frequencies (\%) of Major Depressive Disorder, Generalized Anxiety Disorder, Burnout, and } \\
\text { Zygosity Among 25,378 Swedish Twins, Stratified on Sex }\end{array}$} \\
\hline Variable & Total $N=25,378$ & Women $N=14,114$ & Men $N=11,264$ \\
\hline \multicolumn{4}{|l|}{ Zygosity } \\
\hline MZ & $9,220(36.3)$ & $5,250(37.2)$ & $3,970(35.3)$ \\
\hline DZ same sex & $7,445(29.3)$ & $4,096(29.0)$ & $3,349(29.7)$ \\
\hline DZ opposite sex & $7,883(31.1)$ & $4,400(31.2)$ & $3,483(30.9)$ \\
\hline Unknown & $830(3.3)$ & $368(2.6)$ & $462(4.1)$ \\
\hline \multicolumn{4}{|c|}{ Major depressive disorder } \\
\hline Yes & $2,821(11.1)$ & $2,118(15.0)$ & $703(6.2)$ \\
\hline No & $18,948(74.7)$ & $10,189(72.2)$ & $8,759(77.8)$ \\
\hline Missing & $3,609(14.2)$ & $1,807(12.8)$ & $1,802(16)$ \\
\hline \multicolumn{4}{|c|}{ Generalized anxiety disorder } \\
\hline Yes & $935(3.7)$ & $660(4.7)$ & $275(2.5)$ \\
\hline No & $18,310(72.1)$ & $9,892(70.1)$ & $8,418(74.7)$ \\
\hline Missing & $6,133(24.2)$ & $3,562(25.2)$ & $2,571(22.8)$ \\
\hline \multicolumn{4}{|l|}{ Burnout } \\
\hline Burnout & $4,306(17.0)$ & $3,175(22.5)$ & $1,131(10.0)$ \\
\hline Risk for burnout & $3,783(14.9)$ & $2,331(16.5)$ & $1,452(12.9)$ \\
\hline No burnout & $13,932(54.9)$ & $6,979(49.5)$ & $6,953(61.7)$ \\
\hline Missing & 3,357 (13.2) & $1,629(11.5)$ & $1,728(15.4)$ \\
\hline
\end{tabular}

1. restlessness or feeling keyed up or on edge;

2. being easily fatigued;

3. difficulty concentrating or mind going blank;

4. irritability;

5. muscle tension;

6. sleep disturbance (American Psychiatric Association, 2000).

Burnout was measured with the short form of the Pines Burnout Measure; it correlates strongly (0.90) with the full 21-item Pines Burnout Measure (Hallsten et al., 2005). The scale includes the questions: 'How often during the last 12 months have you felt low?', 'How often during the last 12 months have you felt emotionally exhausted?' and 'How often during the last 12 months have you felt run down?' The answers were given on a 7-point Likert scale ranging from $1=$ never to $7=$ all the time. Cronbach's alpha was 0.89 . The mean burnout score was calculated and a categorical variable was created: no burnout (1-2.99), risk of burnout (3-3.99) and burnout (4-7). Previous studies have used four categories (Takai et al., 2009; Takai et al., 2011); however, as no concordant male DZ twin pairs were present in the highest burnout group, the two highest categories were collapsed.

Zygosity was assessed in STAGE using a set of questions assessing twin pair similarity; this method has been compared with genetic testing in two sub-samples of the twin registry, and proved correct in $98-99 \%$ of the pairs (Lichtenstein et al., 2002). Sex was entered into the analysis as a dichotomous variable.

\section{Analysis}

The biometric model is a type of structural equation model that uses the variance/covariance structure to investigate the genetic and environmental underpinnings of a pheno- type, that is, additive genetics (A), non-additive genetics (D), shared environment (C), and unique environment (E) (Purcell, 2013). Including opposite-sex twins allowed us to test for qualitative sex differences, that is, if the same genes are underpinning the phenotype in women and men, by testing if the genetic correlation can be set to 0.5 in opposite sex twins. As our sample included only twins reared together, C and D could not be tested simultaneously (Rijsdijk \& Sham, 2002). In order to find the best-fitting and most parsimonious model, nested sub-models were tested against the full models or more complex sub-models using likelihood ratio test (Purcell, 2013). As MDD and GAD were binary variables, liability threshold models were used that assumes there is an underlying normally distributed liability to the phenotypes. Answering 'don't know/don't want to answer' was treated as missing values. Polychoric and tetrachoric phenotypic, intrapair, and cross-twin crosstrait correlations were calculated, as they give a first impression of genetic variance and covariance structures in SAS. Analyses was performed using OpenMx software (Boker et al., 2011), run within the R environment (R Development Core Team, 2010).

\section{Univariate Analyses}

To test the assumption that thresholds do not vary between $\mathrm{MZ}$ and DZ twins and between twin 1 and 2 in a pair (randomly assigned), saturated models were specified and compared with a nested model, forcing the thresholds to be equal. As the difference between $\mathrm{MZ}$ and $\mathrm{DZ}$ correlations were somewhat different for men and women, we tested both ACE and ADE models for all phenotypes (Table 2). Models were specified, allowing for both qualitative and quantitative sex differences using five zygosity groups: $M Z$ women, $\mathrm{MZ}$ men, $\mathrm{DZ}$ women, $\mathrm{DZ}$ men, and opposite-sex 


\section{TABLE 2}

Polychoric (Burnout) and Tetrachoric (Major Depressive Disorder and Generalized Anxiety Disorder) Within Pair and Cross-Twin, Cross-Trait Correlations With $95 \%$ Confidence Intervals Among 8,646 Complete Twin Pairs

\begin{tabular}{llcl}
\hline & \multicolumn{3}{c}{ Within-pair correlations } \\
\cline { 2 - 4 } & MDD & GAD & Burnout \\
\hline MZ women & $0.45(0.39-0.50)$ & $0.46(0.34-0.57)$ & $0.34(0.30-0.38)$ \\
MZ men & $0.43(0.32-0.53)$ & $0.35(0.14-0.56)$ & $0.39(0.33-0.45)$ \\
DZ women & $0.12(0.03-0.20)$ & $0.24(0.08-0.39)$ & $0.17(0.12-0.23)$ \\
DZ men & $0.27(0.11-0.42)$ & $0.41(0.15-0.66)$ & $0.07(-0.01-0.15)$ \\
DZ opposite sex & $0.10(0.02-0.18)$ & $0.22(0.08-0.36)$ & $0.10(0.06-0.15)$ \\
& & Cross-twin cross-trait correlations & \\
\cline { 2 - 4 } & MDD and GAD & MDD and Burnout & GAD and Burnout \\
\hline All MZ & $0.37(0.29-0.44)$ & $0.38(0.34-0.42)$ & $0.35(0.29-0.41)$ \\
All DZ & $0.17(0.10-0.25)$ & $0.12(0.08-0.17)$ & $0.09(0.03-0.15)$ \\
\hline
\end{tabular}

Note: $M Z=$ monozygotic, $D Z=$ dizygotic

DZ pairs. As these models are not nested, model selection was based on Bayesian Information Criterion (BIC) values (Markon \& Krueger, 2004; Raftery, 1995). Sub-models were based on the best-fitting model, either ACE or ADE. Sex differences were tested in the full models; first models were tested that only allow for quantitative sex differences, that is, restricting the genetic correlation between the opposite-sex DZ twin pairs to be 0.5 . Subsequently, models forcing the path estimates to be equal for women and men were utilized. AE models were then built, where the D or C parameter was set to zero. E models were then created, where the A parameter was restricted to be zero as well.

\section{Multivariate Analysis}

First, saturated models were used and compared with a nested model to test equal thresholds as in the univariate analysis. In order to investigate the relationship between MDD, GAD, and burnout, we tested three different multivariate models: the Cholesky decomposition, the common factor independent pathway model, and the common factor common pathway model, with one latent factor (Purcell, 2013). In the Cholesky model, three of each of the factors $(\mathrm{A}, \mathrm{C} / \mathrm{D}, \mathrm{E})$ were included. In an independent pathway model, there is one shared $\mathrm{A}, \mathrm{C} / \mathrm{D}$, and $\mathrm{E}$ factor with a path to each phenotype, as well as one separate $A, C / D$, and $\mathrm{E}$ factor per phenotype. As an independent pathway and Cholesky decomposition has the same number of estimated parameters when three variables are used, the best-fitting model was chosen based on BIC value. A common pathway model was then created and compared against the independent pathway model. In a common pathway model, factors load onto a latent common factor that in turn has a path to each phenotype; that is, there is an unmeasured common factor that explains the covariation of the measured phenotypes. It also contains a factor with an independent path to each phenotype. An AE common pathway model was then compared to the full common pathway model. Further, a sub-model removing the phenotype specific to a path to
MDD was compared against the AE model. Finally, an E common pathway model was tested against the AE model without a phenotype specific to a path to MDD.

The study was approved by the regional ethics committee board in Stockholm, Sweden (Dnr: 2009/2053-31/5. Date: 11/02/2010).

\section{Results}

\section{Univariate Analyses}

The models restricting the thresholds to be equal between $\mathrm{MZ}$ and DZ twins and twin 1 and 2 in a pair did not fit significantly worse compared to the saturated models for any of the phenotypes (MDD: $p=.39$, GAD: $p=.30$, burnout: $p=.26)$. We found no statistically significant sex differences; for all phenotypes, removing both qualitative and quantitative sex differences did not significantly worsen the fit of the models (Table 3). Further, removing the D or $\mathrm{C}$ parameters did not result in significantly different fit statistics. However, when removing the A parameter, the models fit significantly worse; hence, AE models with no sex limitations were selected as the final models for all variables (Table 3). All three phenotypes were found to have moderate proportions of the variation explained by additive genetic factors (Table 4).

\section{Multivariate Analysis}

The phenotypic correlations were $0.71(0.69-0.74)$ between MDD and GAD, 0.58 (0.56-0.60) between MDD and burnout, and $0.53(0.50-0.56)$ between GAD and burnout, while cross-twin, cross-trait correlations were similar between all three phenotypes (Table 2). As there were no statistically significant sex differences, two zygosity groups, $\mathrm{MZ}$ and DZ (including opposite-sex DZ twins), were used in the multivariate analysis (Table 3 ). ADE models were used, as the cross-twin, cross-trait correlations for $\mathrm{MZ}$ twins were more than double that of DZ twins (Table 2). The model restricting the thresholds to be equal between $\mathrm{MZ}$ and DZ twins and twin 1 and 2 in a pair did not fit 
TABLE 3

Model Fit Statistics of the Univariate Models for Burnout, Major Depressive Disorder and Generalized Anxiety Disorder and for the Multivariate Models

\begin{tabular}{|c|c|c|c|c|c|c|}
\hline Model & $d f$ & $\mathrm{AlC}$ & $\mathrm{BIC}$ & $-2 \mathrm{LL}$ & $\mathrm{Chi}^{2}$ test & Comparison model \\
\hline \multicolumn{7}{|l|}{ Burnout } \\
\hline ACE GSL & 21,501 & $-4,075.71$ & $-169,078.69$ & $38,926.29$ & & \\
\hline ADE GSL & 21,501 & $-4,075.83$ & $-169,078.81$ & $38,926.17$ & & \\
\hline ADE CSL & 21,502 & $-4,077.71$ & $-169,088.36$ & $38,926.29$ & 0.97 & ADE GSL \\
\hline ADE NSL & 21,505 & $-4,078.67$ & $-169,112.35$ & $38,931.33$ & 0.17 & ADE CSL \\
\hline AE NSL & 21,506 & $-4,080.67$ & $-169,122.02$ & $38,931.33$ & 0.99 & ADE NSL \\
\hline E NSL & 21,507 & $-3,806.48$ & $-168,855.50$ & $39,207.52$ & $<0.01$ & AE NSL \\
\hline \multicolumn{7}{|c|}{ Major depressive disorder } \\
\hline ACE GSL & 21,254 & $-26,297.36$ & $-189,404.81$ & $16,210.64$ & & \\
\hline ADE GSL & 21,254 & $-26,296.02$ & $-189,403.47$ & $16,211.98$ & & \\
\hline ACE CSL & 21,255 & $-26,296.03$ & $-189,411.15$ & $16,213.97$ & 0.07 & ACE GSL \\
\hline ACE NSL & 21,258 & $-26,298.10$ & $-189,436.20$ & $16,217.90$ & 0.27 & ACE CSL \\
\hline AE NSL & 21,259 & $-26,300.10$ & $-189,445.90$ & $16,217.90$ & 1 & ACE NSL \\
\hline E NSL & 21,260 & $-26,143.38$ & $-189,296.88$ & $16,376.62$ & $<0.01$ & AE NSL \\
\hline \multicolumn{7}{|c|}{ Generalized anxiety disorder } \\
\hline ACE GSL & 18,801 & $-30,329.33$ & $-174,611.96$ & $7,272.67$ & & \\
\hline ADE GSL & 18,801 & $-30,326.15$ & $-174,608.78$ & $7,275.85$ & & \\
\hline ACE CSL & 18,802 & $-30,331.26$ & $-174,621.57$ & $7,272.74$ & 0.79 & ACE GSL \\
\hline ACE NSL & 18,805 & $-30,333.95$ & $-174,647.29$ & $7,276.05$ & 0.35 & ACE CSL \\
\hline AE NSL & 18,806 & $-30,334.48$ & $-174,655.49$ & $7,277.52$ & 0.23 & ACE NSL \\
\hline E NSL & 18,807 & $-30,291.11$ & $-174,619.79$ & $7,322.89$ & $<0.01$ & AE NSL \\
\hline \multicolumn{7}{|l|}{ Multivariate } \\
\hline ADE Cholesky & 61,562 & $-64,868.36$ & $-537,307.47$ & $58,255.64$ & & \\
\hline ADE IP & 61,562 & $-64,871.36$ & $-537,310.47$ & $58,252.64$ & & \\
\hline ADE CP & 61,566 & $-64,874.54$ & $-537,344.34$ & $58,257.46$ & 0.31 & ADE IP \\
\hline $\mathrm{AE} \mathrm{CP}$ & 61,570 & $-64,880.25$ & $-537,380.75$ & $58,259.75$ & 0.68 & ADE CP \\
\hline AE CP no A path MDD & 61,571 & $-64,882.24$ & $-537,390.42$ & $58,259.76$ & 0.94 & $\mathrm{AE} C \mathrm{P}$ \\
\hline $\mathrm{E} C P$ & 61,574 & $-64,435.03$ & $-536,966.23$ & $58,712.97$ & $<0.01$ & AE CP no A path MDD \\
\hline
\end{tabular}

Note: Best-fitting model highlighted in bold. $d f=$ degrees of freedom, $-2 \mathrm{LL}=-2$ Log-likelihood, AIC = Akaike's information criterion, $\mathrm{BIC}=$ Bayesian information criterion. Phenotypic variation was decomposed into additive (A) and non-additive (D) genetic variation, and shared (C) and unique (E) environmental variation. GSL = general sex limitation (allows both qualitative and quantitative sex differences). $C S L=$ common sex limitation (allows only quantitative sex differences). NSL $=$ no sex limitation (allows no sex differences). $\mathrm{IP}=$ independent pathway. $\mathrm{CP}=$ common pathway.

\begin{tabular}{|c|c|c|}
\hline \multicolumn{3}{|c|}{$\begin{array}{l}\text { Proportions of Additive Genetic }\left(\mathrm{a}^{2}\right) \text { and Unique } \\
\text { Environmental }\left(\mathrm{e}^{2}\right) \text { Effects From the Best-Fitting Univariate } \\
\text { Models With } 95 \% \text { Confidence Intervals }\end{array}$} \\
\hline & $a^{2}$ & $e^{2}$ \\
\hline Major depressive disorder & $0.45(0.39-0.52)$ & $0.55(0.48-0.61)$ \\
\hline Generalized anxiety disorder & $0.49(0.36-0.61)$ & $0.51(0.39-0.64)$ \\
\hline Burnout & $0.38(0.34-0.43)$ & $0.62(0.57-0.66)$ \\
\hline
\end{tabular}

significantly worse compared to the saturated model $(p=$ .90). The common pathway model was a better fit to data than the independent pathway model and Cholesky decomposition based on BIC and likelihood ratio test (Table 3). Removing the $\mathrm{D}$ factor and the phenotypic specific a path to MDD (which was estimated to zero) did not significantly worsen the fit, hence, this was chosen as the final model (Table 3). The path estimates with $95 \%$ confidence intervals for the model can be found in Figure 1. The covariance components (heritability estimates) can be obtained by squaring the path estimates. Results indicate that there is a latent common factor that is mostly influenced by additive genetic effects (58\%), but also by unique environment (42\%), that explains the covariation between MDD,
GAD, and burnout. This latent common factor explained $77 \%$ of the variation in MDD, $69 \%$ in GAD, and $44 \%$ in burnout. Both burnout and GAD were also found to have phenotype-specific additive genetic effects explaining $11 \%$ of the variance each, while MDD did not have phenotypespecific additive effects. The proportion of the variation that was explained by phenotype-specific unique environmental factors was $45 \%$ for burnout, $23 \%$ for MDD, and 20\% for GAD.

\section{Discussion}

In this cross-sectional twin study, we found that the associations among MDD, GAD, and burnout were consistent with the existence of a single latent common factor influenced mostly by genetics (58\%), but also unique environment $(42 \%)$. For MDD and GAD, the majority of the variation was explained by this common factor $(77 \%$ and $69 \%$, respectively); while for burnout the proportion was lower (44\%). All genetic risk factors for MDD went through the common factor, while GAD and burnout each had $11 \%$ of the variation explained by additive genetic factors unique to each phenotype. The largest proportion of the variation in burnout (45\%) was explained by unique environmental 


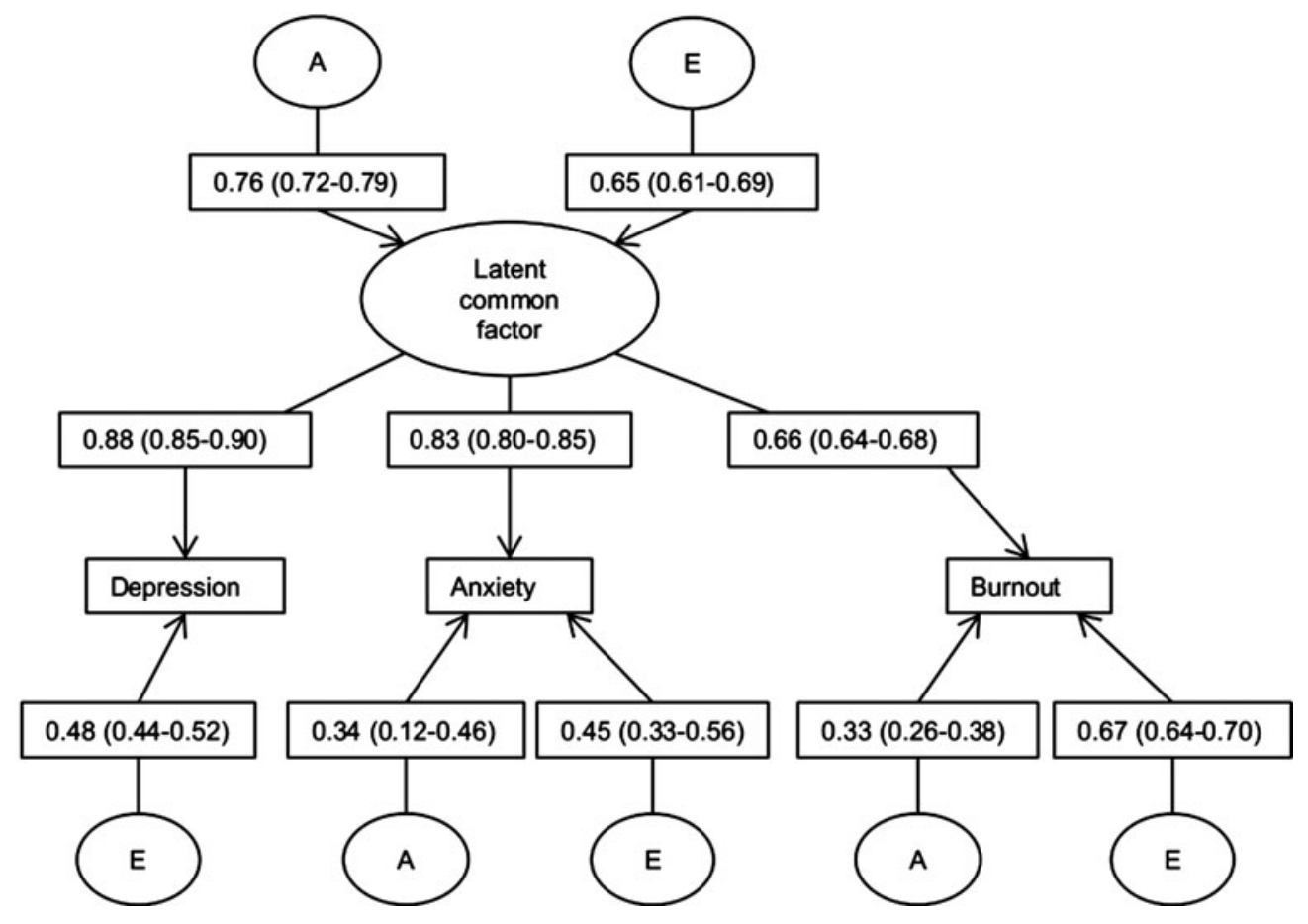

FIGURE 1.

Path estimates for the best-fitting model with $95 \%$ confidence intervals.

factors not shared with MDD and GAD, while for GAD and MDD, environmental factors unique to each phenotype explained $23 \%$ and $20 \%$, respectively.

The fact that a common factor model gave the best fit to the data is in line with a previous twin study, examining the covariation between insomnia, fatigue, and depression, which also found that a common factor, to a large degree influenced by genetic factors, explained the covariation (Hur et al., 2012). Both neuroticism and negative affectivity have been found to be markers for general vulnerability to internalizing disorders (Ormela et al., 2013; Paulus et al., 2015) and a possible explanation is that the latent factor in the present study could represent such an underlying temperament. Neuroticism has been found to correlate genetically with internalizing disorders (Mikolajewski et al., 2013) and share approximately half of its genetic risk factors with depression (Kendler et al., 2006a). However, Kendler et al. (2007) found that only 25\% of the genetic correlation between depression and anxiety were explained by neuroticism, so the latent common factor found in the current study is not likely purely reflecting neuroticism. Many researchers have moved towards looking at underlying temperaments for mental disorders such as depression and anxiety (Brown, 2007). Transdiagnostic interventions for common mental disorders have also been developed and shown symptom improvement (Barlow, 2004; Ejeby et al., 2014). Moreover, the molecular genetic research has begun studying pleiotropy across traditional diagnostic boundaries (Cross-Disorder Group of the Psychiatric Genomics Consortium, 2013).

Whether burnout and depression are separate entities, or whether burnout is a form of depression, is currently under debate (Bianchi et al., 2015b). Our results show that even though burnout shares the majority of its genetic risk with depression, most of the environmental variance and a small amount of genetic variance were unique to burnout. This was despite the fact the Pines Burnout Measure was used, a measure that has been found to be more closely related to depression than the most commonly used measure of burnout, the Maslash Burnout Inventory (Enzmann et al., 1998; Shirom \& Ezrachi, 2003). In a previous study, we also found that both the phenotypic and genetic correlation between burnout, measured with the Pines Burnout Measure, and sick leave due to stress-related mental disorders (0.56) was lower than between burnout and sick leave due to other mental disorders (0.68; Mather, Bergström et al., 2014). The environmental variation unique to burnout could, for example, represent work-related factors such as psychosocial work environment and work-home conflicts, or stressful life events outside work, that have all been shown to be associated with burnout, independent of genetic and shared environmental factors (Blom et al., 2013; Blom et al., 2014; Mather, Blom et al., 2014).

We found high heritability estimates of depression and anxiety compared with previous findings that also found sex differences, while we did not (Hettema et al., 2001; 
Kendler et al., 2006b; Kendler et al., 2007; Middeldorp et al., 2006; Sullivan et al., 2000). Shared environment has been found to have no impact on depression and anxiety (Hettema et al., 2001; Sullivan et al., 2000), while the findings have varied regarding effects of shared environment on burnout (Blom et al., 2012; Middeldorp, Stubble et al., 2005; Middeldorp et al., 2006).

Strengths of the current study include a large sample of twins from the STR, since the register is population based; generalizability of the findings is high for similar age groups. However, since the STAGE questionnaire was so extensive, there are many internal missing values. Due to the amount of missing values and the low prevalence of GAD, this may be why we were unable to find statistically significant sex differences, even though the within-pair correlations indicated there may be differences in heritability in women and men. The fact that the Pines Burnout Measure was used allowed inclusion of all participants. Previous studies have found that burnout is high in groups such as students, athletes, and family caregivers (Dyrbye et al., 2014; Gustafsson et al., 2007; Lindstrom et al., 2010; Takai et al., 2009) and not only in those employed. Moreover, the 'healthy worker effect' often present in burnout studies has been reduced (Schaufeli et al., 2001). Weaknesses include the somewhat low response rate and that burnout was assessed over the last year, while lifetime prevalence of MDD and GAD were measured. Moreover, the measures of MDD and GAD were based on diagnostic criteria, while the Pines Burnout Measure is not a clinical instrument used to assess a diagnosis, but rather mainly a measurement of emotional exhaustion (Shirom \& Ezrachi, 2003). The DSM criteria are meant to be assessed by clinical interview and not as a questionnaire; this may also have affected the sensitivity and specificity of these self-reported measures. There have been varying results when comparing web-based questionnaires with interviews for MDD and GAD (Carlbring et al., 2002; Farvolden et al., 2003; Nguyen et al., 2015). However, self-reported scales measuring depression have been found to capture the genetic variance well when compared with structured clinical interviews, which is considered the gold standard (Foley et al., 2001; Gjerde et al., 2011).

In summary, we found high correlations between MDD, GAD, and burnout (ranging from 0.53 to 0.71 ), which were best explained by a model containing an underlying common factor influenced by genetics (58\%) and unique environment (42\%). All genetic risk factors for MDD were mediated through this factor, while GAD and burnout also had unique genetic risk factors. Burnout was influenced by unique environmental factors to a larger degree than MDD and GAD.

\section{Acknowledgments}

This study was supported by grants from the Swedish Research Council for Health, Working Life and Welfare
(2009-0548), Karolinska Institutet Doctoral student funding (KID), and the Swedish Society of Medicine, and Magnus Bergvall Foundation. The Swedish Twin Registry is supported by Sweden's Department of Higher Education, AstraZeneca, and the Swedish Research Council. The Study of Twin Adults: Genes and Environment (STAGE) was supported by the National Institute of Health, USA, (Grant numbers DK 066134 and CA 085739).

\section{Conflict of Interest}

None.

\section{References}

American Psychiatric Association. (2000). Diagnostic and statistical manual of mental disorders (4th ed.). Washington, DC: Author.

Barlow, D. H. (2004). Psychological treatments. American Psychologist, 59, 869-878.

Bianchi, R., Schonfeld, I. S., \& Laurent, E. (2015a). Burnoutdepression overlap: A review. Clinical Psychology Review, $36,28-41$.

Bianchi, R., Schonfeld, I. S., \& Laurent, E. (2015b). Is it time to consider the 'Burnout Syndrome' A distinct illness? Frontiers in Public Health, 3, 158.

Blom, V., Bergstrom, G., Hallsten, L., Bodin, L., \& Svedberg, P. (2012). Genetic susceptibility to burnout in a Swedish twin cohort. European Journal of Epidemiology, 27, 225-231.

Blom, V., Bodin, L., Bergstrom, G., Hallsten, L., \& Svedberg, P. (2013). the importance of genetic and shared environmental factors for the associations between job demands, control, support and burnout. PLoS One, 8, e75387.

Blom, V., Sverke, M., Bodin, L., Bergstrom, G., Lindfors, P., \& Svedberg, P. (2014). Work-home interference and burnout: A study based on Swedish twins. Journal of Occupational and Environmental Medicine, 56, 361-366.

Boker, S., Neale, M., Maes, H., Wilde, M., Spiegel, M., Brick, T., ... Fox, J. (2011). OpenMx: An open source extended structural equation modeling framework. Psychometrika, 76, 306-317.

Brown, T. A. (2007). Temporal course and structural relationships among dimensions of temperament and DSM-IV anxiety and mood disorder constructs. Journal of Abnormal Psychology, 116, 313-328.

Carlbring, P., Forslin, P., Ljungstrand, P., Willebrand, M., Strandlund, C., Ekselius, L., \& Andersson, G. (2002). Is the internet-administered CIDI-SF equivalent to a clinicianadministered SCID Interview. Cognitive Behaviour Therapy, 31, 183-189.

Cross-Disorder Group of the Psychiatric Genomics Consortium. (2013). Identification of risk loci with shared effects on five major psychiatric disorders: A genome-wide analysis. Lancet, 381, 1371-1379.

Ding, Y. W., Qu, J. W., Yu, X. S., \& Wang, S. (2014). The mediating effects of burnout on the relationship between anxiety symptoms and occupational stress among community 
healthcare workers in China: A cross-sectional study. PLoS One, 9, e107130.

Dyrbye, L. N., West, C. P., Satele, D., Boone, S., Tan, L., Sloan, J., \& Shanafelt, T. D. (2014). Burnout Among U.S. medical students, residents, and early career physicians relative to the general U.S. population. Academic Medicine, 89, 443451.

Ejeby, K., Savitskij, R., Ost, L. G., Ekbom, A., Brandt, L., Ramnero, J., ... Backlund, L. G. (2014). Randomized controlled trial of transdiagnostic group treatments for primary care patients with common mental disorders. Family Practice, 31, 273-280.

Enzmann, D., Schaufeli, W. B., Janssen, P., \& Rozeman, A. (1998). Dimensionality and validity of the Burnout Measure. Journal of Occupational and Organizational Psychology, 71, 331-351.

Farvolden, P., McBride, C., Bagby, R. M., \& Ravitz, P. (2003). A web-based screening instrument for depression and anxiety disorders in primary care. Journal of Medical Internet Research, 5, e23.

First, M. B., Spitzer, R. L., Gibbon, M., Williams, J. B. W., \& Benjamin, L. (1996). Structured clinical interview for DSM$I V$-patients edition (with psychotic screen, version 2.0). New York: Biometrics Research Department, New York State Psychiatric Institute.

Foley, D. L., Neale, M. C., \& Kendler, K. S. (2001). Genetic and environmental risk factors for depression assessed by subject-rated Symptom Check List versus Structured Clinical Interview. Psychological Medicine, 31, 1413-1423.

Furberg, H., Lichtenstein, P., Pedersen, N., Thornton, L., Bulik, C., Lerman, C., \& Sullivan, P. (2008). The STAGE cohort: A prospective study of tobacco use among Swedish twins. Nicotine \& Tobacco Research, 10, 1727-1735.

Gatt, J. M., Burton, K. L., Williams, L. M., \& Schofield, P. R. (2015). Specific and common genes implicated across major mental disorders: A review of meta-analysis studies. Journal of Psychiatric Research, 60, 1-13.

Gjerde, L. C., Røysamb, E., Czajkowski, N., ReichbornKjennerud, T., Ørstavik, R. E., Kendler, K.S., \& Tambs, K. (2011). Strong genetic correlation between interviewassessed internalizing disorders and a brief self-report symptom scale. Twin Research and Human Genetics, 14, 6472.

Global Burden of Disease Study 2013 Collaborators. (2015). Global, regional, and national incidence, prevalence, and years lived with disability for 301 acute and chronic diseases and injuries in 188 countries, 1990-2013: A systematic analysis for the Global Burden of Disease Study 2013. Lancet, 386, 743-800.

Gustafsson, H., Kentta, G., Hassmen, P., \& Lundqvist, C. (2007). Prevalence of burnout in competitive adolescent athletes. Sport Psychologist, 21, 21-37.

Hallsten, L., Josephson, M., \& Torgén, M. (2005). Performancebased self-esteem - A driving force in burnout processes and its assessment. Stockholm: National Institute for Working Life.
Hettema, J., Neale, M., \& Kendler, K. (2001). A review and meta-analysis of the genetic epidemiology of anxiety disorders. American Journal of Psychiatry, 158, 1568-1578.

Hur, Y. M., Burri, A., \& Spector, T. D. (2012). The genetic and environmental structure of the covariation among the symptoms of insomnia, fatigue, and depression in adult females. Twin Research and Human Genetics, 15, 720-726.

Kendler, K. S., Gardner, C. O., Gatz, M., \& Pedersen, N. L. (2007). The sources of co-morbidity between major depression and generalized anxiety disorder in a Swedish national twin sample. Psychological Medicine, 37, 453-462.

Kendler, K. S., Gatz, M., Gardner, C. O., \& Pedersen, N. L. (2006a). Personality and major depression: A Swedish longitudinal, population-based twin study. Archives of General Psychiatry, 63, 1113-1120.

Kendler, K. S., Gatz, M., Gardner, C. O., \& Pedersen, N. L. (2006b). A Swedish national twin study of lifetime major depression. Am J Psychiatry, 163, 109-114.

Kendler, K. S., Neale, M. C., Kessler, R. C., Heath, A. C., \& Eaves, L. J. (1992). Major depression and generalized anxiety disorder. Same genes, (partly) different environments? Archives of General Psychiatry, 49, 716-722.

Lichtenstein, P., de Faire, U., Floderus, B., Svartengren, M., Svedberg, P., \& Pedersen, N. L. (2002). The Swedish Twin Registry: A unique resource for clinical, epidemiological and genetic studies. Journal of Internal Medicine, 252, 184205.

Lichtenstein, P., Sullivan, P. F., Cnattingius, S., Gatz, M., Johansson, S., Carlstrom, E., ... Pedersen, N. L. (2006). The Swedish Twin Registry in the third millennium: An update. Twin Research and Human Genetics, 9, 875-882.

Lindstrom, C., Aman, J., \& Norberg, A. L. (2010). Increased prevalence of burnout symptoms in parents of chronically ill children. Acta paediatrica, 99, 427-432.

Magnusson, P. K., Almqvist, C., Rahman, I., Ganna, A., Viktorin, A., Walum, H., ... Lichtenstein, P. (2013). The Swedish Twin Registry: establishment of a biobank and other recent developments. Twin Research and Human Genetics, 16, 317-329.

Markon, K. E., \& Krueger, R. F. (2004). An empirical comparison of information-theoretic selection criteria for multivariate behavior genetic models. Behavior Genetics, 34, 593-610.

Maslach, C., Schaufeli, W. B., \& Leiter, M. P. (2001). Job burnout. Annual Review of Psychology, 52, 397-422.

Mather, L., Bergström, G., Blom, V., \& Svedberg, P. (2014). The covariation between burnout and sick leave due to mental disorders is explained by a shared genetic liability: A prospective Swedish twin study with a five-year follow-up. Twin Research and Human Genetics, 17, 535544.

Mather, L., Blom, V., \& Svedberg, P. (2014). Stressful and traumatic life events are associated with burnout-a crosssectional twin study. International Journal of Behavioral Medicine, 21, 899-907.

Middeldorp, C. M., Cath, D. C., \& Boomsma, D. I. (2006). A twin-family study of the association between employment, 
burnout and anxious depression. Journal of Affective Disorders, 90, 163-169.

Middeldorp, C. M., Cath, D. C., Van Dyck, R., \& Boomsma, D. I. (2005). The co-morbidity of anxiety and depression in the perspective of genetic epidemiology. A review of twin and family studies. Psychological Medicine, 35, 611-624.

Middeldorp, C. M., Stubble, J. H., Cath, D. C., \& Boomsma, D. I. (2005). Familial clustering in burnout: A twin-family study. Psychological Medicine, 35, 113-120.

Mikolajewski, A. J., Allan, N. P., Hart, S. A., Lonigan, C. J., \& Taylor, J. (2013). Negative affect shares genetic and environmental influences with symptoms of childhood internalizing and externalizing disorders. Journal of Abnormal Child Psychology, 41, 411-423.

Nguyen, D. P., Klein, B., Meyer, D., Austin, D. W., \& Abbott, J. A. (2015). The diagnostic validity and reliability of an internet-based clinical assessment program for mental disorders. Journal of Medical Internet Research, 17, e218.

Nyklicek, I., \& Pop, V. J. (2005). Past and familial depression predict current symptoms of professional burnout. Journal of Affective Disorders, 88(1), 63-68.

Ormela, J., Jeronimusa, B. F., Kotovc, R., Riesea, H., Bosa, E. H., Hankind, B., ... Oldehinkela, A. J. (2013). Neuroticism and common mental disorders: Meaning and utility of a complex relationship. Clinical Psychology Review, 33, 686-697.

Paulus, D. J., Talkovsky, A. M., Heggeness, L. F., \& Norton, P. J. (2015). Beyond negative affectivity: A hierarchical model of global and transdiagnostic vulnerabilities for emotional disorders. Cognitive Behaviour Therapy, 44, 389-405.

Pines, A. M., \& Aronson, E. (1988). Career burnout: Causes and cures. New York: Free Press.

Pines, A. M., Aronson, E., \& Kafry, D. (1981). Burnout: from tedium to personal growth. New York: The Free Press.
Purcell, S. (2013). Statistical methods in behavioral genetics. In R. Plomin, J. C. DeFries, V. S. Knopik \& J. M. Neiderhiser (Eds.), Behavioral genetics (6th ed., pp. 357-411). New York: Worth Publishers.

R Development Core Team. (2010). R foundation for statistical computing. Vienna, Austria: Author.

Raftery, A. E. (1995). Bayesian model selection in social research. Sociological Methodology, 25, 111-163.

Rijsdijk, F., \& Sham, P. (2002). Analytic approaches to twin data using structural equation models. Briefings in Bioinformatics, 3, 119-133.

Schaufeli, W. B., Bakker, A. B., Hoogduin, K., Schaap, C., \& Klader, A. (2001). On the clinical vailidty of the Maslach burnout inventory and the burnout measure. Psychology and Health, 16, 565-582.

Shirom, A., \& Ezrachi, Y. (2003). On the discriminant validity of burnout, depression and anxiety: A re-examination of the Burnout Measure. Anxiety, Stress and Coping, 16, 8397.

Sullivan, P. F., Neale, M. C., \& Kendler, K. S. (2000). Genetic epidemiology of major depression: Review and metaanalysis. American Journal of Psychiatry, 157, 1552-1562.

Takai, M., Takahashi, M., Iwamitsu, Y., Ando, N., Okazaki, S., Nakajima, K., ... Miyaoka, H. (2009). The experience of burnout among home caregivers of patients with dementia: Relations to depression and quality of life. Archives of Gerontology and Geriatrics, 49, e1-e5.

Takai, M., Takahashi, M., Iwamitsu, Y., Oishi, S., \& Miyaoka, H. (2011). Subjective experiences of family caregivers of patients with dementia as predictive factors of quality of life. Psychogeriatrics 11, 98-104.

Toker, S., Shirom, A., Shapira, I., Berliner, S., \& Melamed, S. (2005). The association between burnout, depression, anxiety, and inflammation biomarkers: C-reactive protein and fibrinogen in men and women. Journal of Occupational Health Psychology, 10, 344-362. 